\title{
Molecular dynamics investigation of conversion methods for excess adsorption amount of shale gas
}

\author{
Wenbin Jiang ${ }^{\mathrm{a}}$, Mian Lin ${ }^{\mathrm{a}, \mathrm{b}, *}$ \\ a Institute of Mechanics, Chinese Academy of Sciences, Beijing, 100190, China \\ b University of Chinese Academy of Sciences, Beijing, 100190, China
}

\section{A R T I C L E I N F O}

\section{Keywords:}

Shale gas

Adsorbed-phase density

Excess adsorption

Absolute adsorption

Molecular dynamics

\begin{abstract}
A B S T R A C T
In investigations of shale gas found within shale formations, the conversion of the experimentally determined excess adsorption amount to the absolute amount is crucial to the interpretation of experimental adsorption data. In our study, the excess isotherms of a shale sample from the Longmaxi Formation in China are converted to absolute ones using existing methods. Keeping in mind that the results differ widely across methods and that the accuracies of these methods are poorly understood, we take a first step towards revealing and evaluating the possible discrepancies. We construct a molecular dynamics (MD) model considering methane adsorption in nanometer channels of different widths, and the model is able to calculate the excess and absolute adsorption amounts. Our simplified MD model is proved to be able to reproduce the excess adsorption isotherms of the shale sample through comparison with experimental data. We also evaluate existing conversion methods for calculation of the excess adsorption amount based on MD results. The methods that use predetermined adsorbedphase density values usually underestimate the absolute amount. Further, the modified Langmuir and Töth equation methods underestimate results under most conditions. The relative error is large at higher pressure, higher temperature, and in smaller channels, and it can be as large as45\%. Our study will serve to remind researchers about uncertainty of the results these methods, which can be useful in application.
\end{abstract}

\section{Introduction}

Shale gas is a natural gas that is found trapped within shale formations. Shale gas has attracted increasing interest as an important natural gas source, particularly in the context of global energy requirements. In this context, high-pressure mercury intrusion and scanning electron microscopy (SEM) have mostly been utilized to determine that nanometer pores are dominant in shale gas reservoirs (Loucks et al., 2009; Nelson, 2009; Curtis et al., 2012; Chen et al., 2013). The main constituent of shale gas is methane. Shale gas exists in three forms, namely, free gas, adsorbed gas and dissolved gas in nanometer pores, and the first two forms are predominant (Liu et al., 2015; Sigal et al., 2015; Gao, 2016; Ahmed and Meehan, 2016). The adsorbed gas content may exceed $50 \%$ of the total shale gas content (Lu et al., 1995; Ambrose et al., 2012). Therefore, precisely determining the amount of adsorbed gas and its variation with respect to pressure is critical for the evaluation of shale gas in place (GIP) and the estimated ultimate recovery.

Isothermal adsorption experiments form one of the most commonly used means to measure the shale gas adsorption amount (Ji et al., 2012;
Zhang et al., 2012; Rexer et al., 2013, 2014; Santos and Akkutlu, 2013). In these experiments, at every equilibrium pressure point, the difference of pressures in the sample chamber before and after equilibrium is measured. The free gas content reduction can be calculated based on the pressure reduction using the equation of state. According to the law of mass conservation, the measured gas content increase is equal to the free gas content reduction. However, this quantity only accounts for the extra gas content in consideration of the occurrence of adsorption in comparison with the absence of adsorption, and it is called the excess adsorption amount. In many flow models considering the influence of adsorption (Cui et al., 2009; Civan et al., 2010; Li et al., 2014a, 2014b; Naraghi and Javadpour, 2015), researchers utilize the total gas content in the adsorbed layer, namely, the absolute adsorption amount. Thus, the measured excess amount needs to be converted to the absolute one. The following general relationship exists between these two physical quantities (Rexer et al., 2013):

$q_{a b}=q_{e x} /\left(1-\rho_{\text {free }} / \rho_{a d}\right)$

Here, $q_{e x}$ denotes the excess amount, $q_{a b}$ the absolute amount, $\rho_{\text {free }}$ the free gas (bulk phase) density, and $\rho_{a d}$ the adsorbed phase density.

\footnotetext{
* Corresponding author. Institute of Mechanics, Chinese Academy of Sciences, Beijing, 100190, China.

E-mail address: linmian@imech.ac.cn (M. Lin).
} 
When the pressure is low, $\rho_{\text {free }}$ is so small compared with $\rho_{a d}$ that there is no obvious difference between $q_{e x}$ and $q_{a b}$. It is well known that when the pressure is lower than $1 \mathrm{MPa}$, this difference can be neglected (Rexer et al., 2013). For higher pressure, the direct use of $q_{e x}$ instead of $q_{a b}$ leads to underestimation of the adsorption amount.

The key quantity involved in the abovementioned conversion is $\rho_{a d}$. However, there is no standard value for this quantity. In this context, existing conversion methods can be divided into two categories: In category 1 methods, $\rho_{a d}$ is fixed to a predetermined value, usually between $0.373 \mathrm{~g} / \mathrm{cc}$ (the van der Waals co-volume constant, $b$; Hall et al., 1994; Ambrose et al., 2012) and $0.423 \mathrm{~g} / \mathrm{cc}$ (the density of liquid methane at its boiling point (Gasparik et al., 2012; Rexer et al., 2013; Clarkson and Haghshemas, 2013)). In category 2 methods, $\rho_{a d}$ is determined by fitting the excess isotherm to a modified equation (Gasparik et al., 2012; Mouahid et al., 2012; Rexer et al., 2013; Yu et al., 2015). The modified equation introduces $\rho_{a d}$ into the original equation for the absolute isotherms. Importantly, we note that $\rho_{a d}$ varies from sample to sample (Gasparik et al., 2012; Rexer et al., 2013). For example, the modified Langmuir equation for excess isotherms can be expressed as follows (Gasparik et al., 2012; Mouahid et al., 2012; Rexer et al., 2013):

$q_{e x}=\left(\frac{V_{L} p}{p_{L}+p}\right)\left(1-\frac{\rho_{\text {free }}}{\rho_{\text {ad }}}\right)$

where $V_{L}$ represents the maximum adsorbed amount and $P_{L}$ the Langmuir pressure. The first term on the right-hand side represents the standard Langmuir equation for absolute isotherms. When the Töth equation (Toth, 1995) is applied to absolute isotherms, the corresponding modified equation for excess isotherms is as follows:

$q_{\text {ex }}=\frac{V_{T} p}{\left[p_{T}^{n}+p^{n}\right]^{1 / n}}\left(1-\frac{p_{\text {free }}}{p_{a d}}\right)$

where $V_{T}$ and $P_{T}$ are similar in meaning to $V_{L}$ and $P_{L}$, respectively, and $n$ denotes an exponent that relates to the heterogeneity of the adsorbent surface. The first term on the right-hand side represents the standard Töth equation.

In this study, the $30{ }^{\circ} \mathrm{C}$ excess isotherm of a shale sample from the Longmaxi Formation in Sichuan, China (Yu et al., 2015) is converted into absolute isotherms using the two abovementioned categories of methods (more details are presented in Appendix A.). With the application of methods of the first category, the absolute amounts decline with pressure under high-pressure conditions (Fig. A1). With the applications of the methods of the second category, the fitted $\rho_{a d}$ values from the modified Langmuir and Töth equations are different and this discrepancy is about $10 \%$ (Table A3), and the converted absolute isotherms are obviously different (Fig. A2). Here, we remark that the Langmuir equation is widely used in studies relating to shale gas adsorption (Chen, 2016; Song et al., 2016). However, it has also been found that Langmuir equation is unsuitable for certain shale samples (Chareonsuppanimit et al., 2012; Yang et al., 2013). Therefore, it cannot be predetermined as to which modified equation is more suitable for a given excess isotherm. Therefore, the selection of different conversion methods may yield uncertainty, and this aspect needs to be further evaluated.

Isothermal adsorption experiments only provide integral quantities of samples, and they cannot clearly distinguish between the free and adsorbed phases in nanometer pore; the adsorbed-phase density cannot be determined in these experiments. In recent years, scientists have used molecular dynamics (MD) or grand canonical Monte Carlo (GCMC) simulation to obtain the gas density distribution in a single nanometer pore. In this regard, Ambrose et al. (2012) used graphite layers to represent kerogen walls in shale and simulated methane adsorption in a nanometer slit-pore at $80{ }^{\circ} \mathrm{C}, 20.98 \mathrm{MPa}$. The authors determined that there is an obvious adsorbed layer in the area close to the wall and that the adsorbed methane density is $0.34 \mathrm{~g} / \mathrm{cc}$.
Meanwhile, Mosher et al. (2013) used GCMC to simulate methane adsorption in 0.4-9-nm-wide channels at 1-12 MPa to investigate variations in the adsorption characteristics. Further, Wu et al. (2015) used $\mathrm{MD}$ to simulate methane adsorption and displacement by $\mathrm{CO}_{2}$ in carbon nanometer channels. The wall materials were subsequently changed to other inorganic materials. Sharma et al. (2015) used GCMC and MD to simulate methane and ethane adsorptions and diffusions in montmorillonite pores. Sui et al. (2015) investigated the influence of water molecules on methane adsorption in montmorillonite pores by means of MD, and Li et al. (2016) simulated methane adsorptions in graphite and calcite pores, and the influence of different channel widths were investigated and the variations in density and pressure profiles in the channels were obtained. From the above researches, it can be observed that methane density distributions in nanometer pores can be acquired via MD/GCMC. Therefore, this approach is promising to separate the adsorbed phase from the free phase, and determine the absolute adsorption amount. Subsequently, the commonly used conversion methods for the excess amount can be suitably evaluated.

In this study, we utilize MD to simulate methane adsorption in nanometer graphite channels. The excess and absolute adsorption amounts are calculated based on the MD simulation results. The applicability and possible deviation of existing methods under conditions of different channel widths, pressure and temperature are subsequently evaluated. This paper is organized as follows: Our methodology is introduced in Section 2, while results and discussions are presented in Section 3. Section 4 concludes the paper.

\section{Methodology}

\subsection{Molecular dynamics simulation}

\subsubsection{Cell construction}

A graphite nanometer channel is a simplified representation of a nanometer pore in shale (Ambrose et al., 2012; Liu et al., 2016), and it consists of upper and lower three-layer-graphite walls and a central void space. The bond length between two neighboring carbon atoms (C_g) in the same graphite layer is $1.42 \AA$, and the interlamellar spacing of graphite is $3.35 \AA$. This structure is extended periodically along the horizontal directions. The carbon atoms occupy the sphere space with a diameter of $\sigma_{c g}$ which is their Lennard-Jones diameter. The distance between the centers of the nearest upper and lower layers is $H$. The width of the channel $D$ is therefore the difference between $H$ and $\sigma_{c g}$ (Fig. 1). The united-atom (UA) methane molecules ( $\mathrm{C}_{-} \mathrm{m}$ ) are used here.

\subsubsection{Cell dimension}

To ensure the stability of the estimated statistical quantities, such as temperature and pressure, the number of $C_{-} \mathrm{m}$ molecules in the cell should exceed 500 . When the channel width, temperature and expected pressure are given, the horizontal dimensions of a channel determine the number of $\mathrm{C}_{-} \mathrm{m}$ molecules in it. Therefore, in order to consider different temperature and pressure conditions, the horizontal dimensions should be free to expand or shrink. In this study, a routine is designed to copy a single graphite lattice in $\mathrm{X}$ and $\mathrm{Y}$ directions to achieve this point. Different channel widths are realized by adjusting $\mathrm{Z}$ coordinates of graphite layers.

\subsubsection{Initial positions of methane molecules}

A certain number of methane molecules should be placed in the channel in the initial computation step. If methane molecules lie beyond the void space or are too close to each other, the simulation will diverge rapidly. An intuitive approach is to arrange molecules regularly in the void space. However, when the void space is non-orthogonal and the methane is very dense (under high pressure conditions), it can be very cumbersome to calculate the molecule positions. In this study, we propose an algorithm to randomly distribute C_m molecules. The judging criteria for a valid position include: 1) the molecule cannot go 


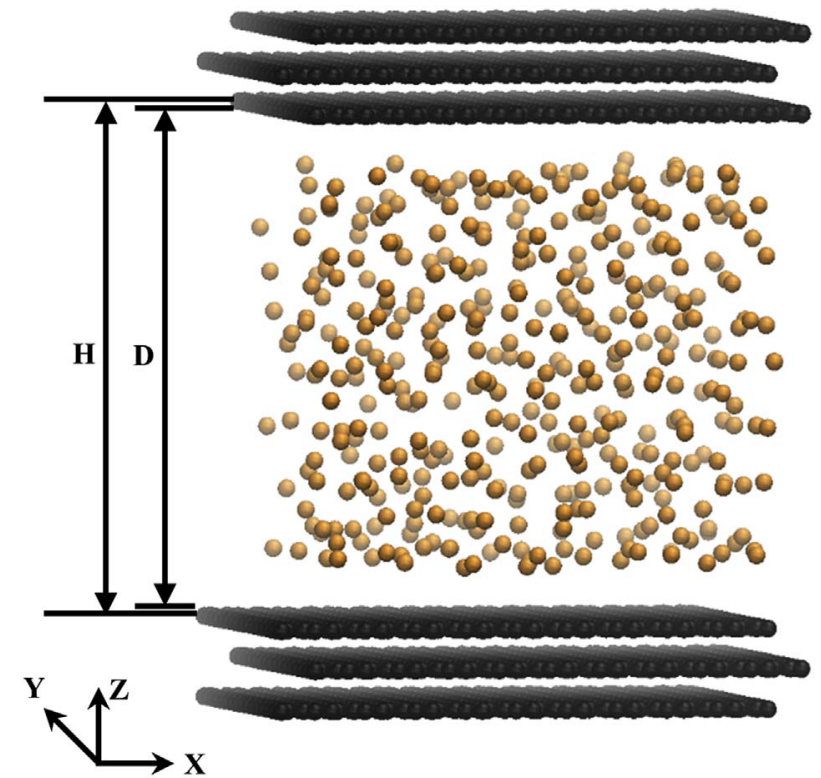

Fig. 1. Simulation cell used in this study (the black spheres represent $C_{-} g$ and the yellow ones represent $\mathrm{C}_{-} \mathrm{m}$ ). (For interpretation of the references to colour in this figure legend, the reader is referred to the web version of this article.)

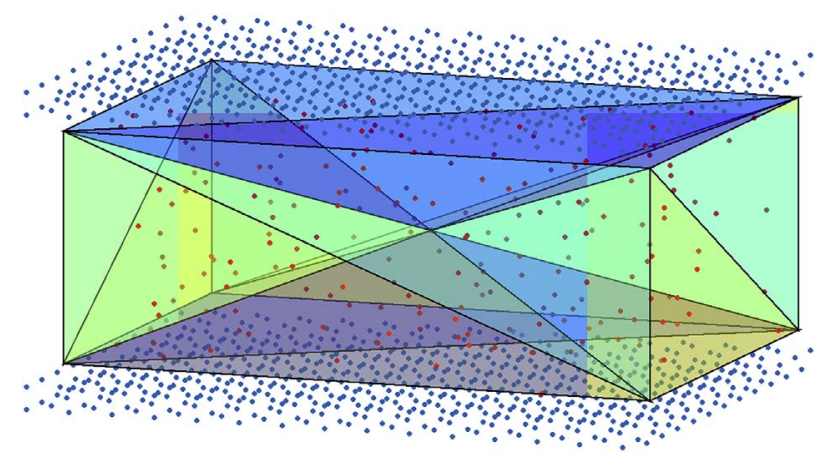

Fig. 2. Random distribution of methane molecules in simulated channel (D $=3.8 \mathrm{~nm}$, $\left.21.96 \mathrm{MPa}, 80^{\circ} \mathrm{C}\right)$.

beyond the void space, namely, the inserted C_m molecule and six outside surfaces of the void space will form six tetrahedrons (Fig. 2), and if the inserted molecule is inside the void space the sum of volumes of these tetrahedrons should be equal to the volume of the void space; 2) the distances between the newly inserted molecule and other already-added ones should be larger than the Lennard-Jones diameter, $\sigma_{c m}$. Fig. 2 shows the distribution of 1295 methane molecules in a 3.8nm-wide channel with the use of this algorithm.

\subsubsection{Simulation parameters}

In the channel, the adsorption is controlled by the methane-methane and methane-graphite van der Waals forces (Ambrose et al., 2012; Mosher et al., 2013). In this study, we adopt the molecular dynamics code, LAMMPS (Plimpton, 1995), and we use the following LennardJones potential function:

$U(r)=4 \varepsilon\left[\left(\frac{\sigma}{r}\right)^{12}-\left(\frac{\sigma}{r}\right)^{6}\right]$

where $\varepsilon$ and $\sigma$ represent the van der Waals force parameters and $r$ the distance of the molecule pair. Optimized Potentials for Liquid Simulations-United Atom (OPLS-UA) force field is utilized, and the values of the above parameters are listed in Table 1. The Lorentz-Berthelot mixing rules: $\sigma_{i j}=\left(\sigma_{i i}+\sigma_{j j}\right) / 2$ and $\varepsilon_{i j}=\sqrt{\varepsilon_{i i} \varepsilon_{j j}}$ are used to determine forces between different types of atoms (Ambrose et al., 2012). Here, $\sigma_{i j}$
Table 1

Parameters of potential functions.

\begin{tabular}{lll}
\hline Molecule & $\varepsilon / \mathrm{kcal} / \mathrm{mol}$ & $\sigma / \mathrm{nm}$ \\
\hline C_g(Graphite) & 0.0556 & 0.34 \\
C_m(Methane) & 0.2940 & 0.373 \\
\hline
\end{tabular}

Table 2

Computation parameters.

\begin{tabular}{ll}
\hline Parameter & Value \\
\hline Relaxation time & $0.3 \mathrm{ps}$ \\
Computation time step & $0.003 \mathrm{ps}$ \\
Number of steps to equilibrium & 167000 \\
Total steps & 500000 \\
\hline
\end{tabular}

and $\varepsilon_{i j}$ represent the van der Waals force parameters for a methane molecule and a carbon atom. The graphite acts as the pore wall and the carbon atoms are frozen during simulation. The NVT ensemble and Nosé-Hoover temperature-controlling algorithm are adopted with the parameters in Table 2. After equilibrium, the density distribution along the $\mathrm{Z}$ direction is calculated every 1000 steps as follows: the channel is divided into bins with intervals of $0.02 \mathrm{~nm}$ along the vertical direction, and the time-averaged methane amount in each bin is gathered and the density in each bin is determined by dividing the volume of the bin. The pressure is the pore pressure for the bulk phase.

\subsection{Adsorption amount calculation}

For convenience of analysis, the adsorption amount considered in this study is actually the amount per unit surface area $\left(\mathrm{g} / \mathrm{m}^{2}\right)$.

\subsubsection{Calculation of excess adsorption amount}

In order to describe the procedure to calculate the excess amount, we analyze the simulated methane density profile in a 3.8 -nm-wide channel at $80{ }^{\circ} \mathrm{C}$ and $21.96 \mathrm{MPa}$ as an example (Fig. 3). The void space in the channel can be separated into ten 0.38-nm-wide vertical layers. The continuous methane density curve (blue line) in each layer is partitioned and summarized to discrete density curve (red line in Fig. 3) (Ambrose et al., 2012). It can be observed that the densities of the layers close to the wall are greater than those of the central layers. The gas phase in the middle layers can be referred to as the bulk phase. After adding the excessive densities above the bulk phase in layers nonadjacent to the walls into the layers closest to the walls, the discrete

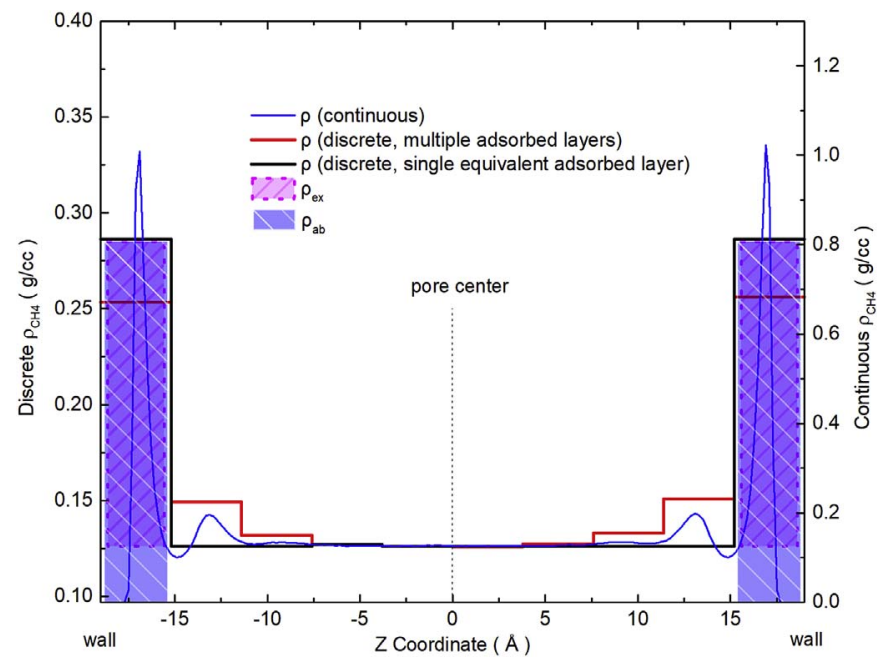

Fig. 3. Methane density profiles in the nanometer channel. 
density curve (red line) can be converted to that represented by the black line. Two equivalent adsorbed layers are formed. The excess adsorption part is denoted by the purple patches with the dashed borders, and this part only accounts for the component that exceeds the bulk phase (central) gas density. The adsorption amount is the product of density and the thickness of one layer, which is $0.38 \mathrm{~nm}$.

\subsubsection{Calculation of absolute adsorption amount}

The absolute adsorption amount can be obtained by means of the two following approaches: 1 ) in an MD simulation, the total amount of methane molecules in the nearest layer from the wall is the absolute adsorption amount, and this is denoted by the blue patches in Fig. 3. 2) Besides the above result directly derived from MD simulation, the absolute amount can also be converted using the two abovementioned categories of methods from the excess amount obtained by MD simulation. The results obtained with the first approach can be used as a criterion to evaluate the discrepancies of results obtained with the second approach.

\section{Results and discussion}

\subsection{Applicability of MD simulations to evaluate conversion methods for} shale gas samples

\subsubsection{Validity of methane-methane interaction parameters}

In order to confirm the validity of the methane-methane force parameters, the relationship between density and pressure is calculated in the free gas state without pore walls. The NPT ensemble is used in this situation, that is, the volume of the simulation cell is allowed to expand or shrink. The density is determined based on the balanced volume at a certain temperature and pressure. This simulation result is compared with those of Ambrose et al.'s (2012) work and the NISTSUPERTRAPP database. From Fig. 4, we observe that this difference is very small, which reveals that the methane-methane force parameters are suitable for application in this study.

\subsubsection{Comparison of $M D$ simulations and experiments}

From a MD perspective, the free phase is mainly controlled by the methane-methane force, while the adsorption is mainly controlled by the methane-wall interactions. The verification of the latter set of interactions is the base of the following analyses.

In an actual situation, the experimental shale sample contains both inorganic and organic matters (pore wall), and the void space is tortuous, rough and irregular. Therefore, it is currently impossible to construct a simulation cell that has the same content and structure as

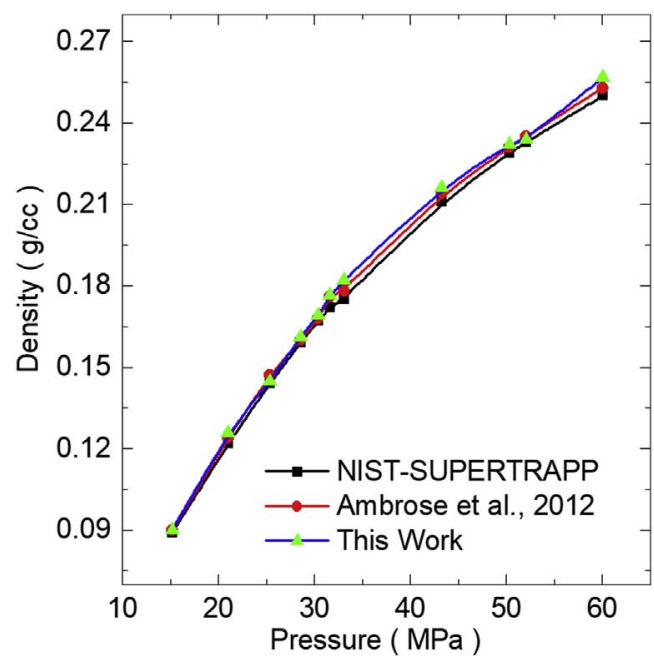

Fig. 4. Demonstration of validity of relationship between methane density and pressure. that of the experimental sample. Considering that the aim of this study is to employ MD simulations to evaluate the discrepancies between different conversion methods, and not to exactly predict the adsorption ability under different conditions, simplified MD simulations that can reproduce experimental excess isotherms are acceptable.

Other than at $30{ }^{\circ} \mathrm{C}$, excess adsorption isotherms of the sample from Longmaxi Formation at three other temperatures have also been measured by Yu et al. (2015). The isotherms were measured by Yu et al. (2015) by means of a Rubotherm IsoSORP Gravimetric Sorption Analyzer. The $20 \mathrm{~g}$ sample, the screening size of which is between 0.38 and $0.83 \mathrm{~mm}$, was dried for over $24 \mathrm{~h}$ at $105{ }^{\circ} \mathrm{C}$ and then degassed by vacuum for $4 \mathrm{~h}$ before the experiment. The BET-specific surface area of the sample was measured as $17.8 \mathrm{~m}^{2} / \mathrm{g}$ by means of a QuadraSorb Station 3 nitrogen gas adsorption surface area analyzer. In this study, simulations were performed at the same temperatures, and the excess adsorption densities were determined by the method introduced in Section 2.2.1. After multiplication by the thickness of a single layer $(0.38 \mathrm{~nm})$ and the specific surface area of the sample, the excess adsorption amount was determined. A comparison of the results obtained by MD simulation and experiments indicates consistency of the trend that excess isotherms first increase then decline with pressure is consistent (Fig. 5). Another trend that the turning pressure points of isotherms increase with temperatures is also consistent between experiment and simulation. Therefore, the constructed MD simulation model can suitably capture the methane-wall interactions well, and can be used as a criterion to evaluate the different conversion methods for excess isotherms of shale gas samples.

\subsection{Evaluation of conversion methods and sensitivity analyses}

Based on the same excess adsorption isotherm, different absolute isotherms can be acquired by use of different conversion methods. The MD simulation result provides a clear definition of absolute adsorption, and it does not depend on relationships such as Eq. (1) and the adsorbed-phase density. Therefore, it can be used as a criterion to evaluate the results obtained by the two abovementioned categories of methods. In addition, MD simulations can be conducted under a broader range of conditions, and they provide possibilities for more comprehensive evaluations and sensitivity analyses. In this study, we performed MD evaluations for different channel widths, pressures and temperatures.

\subsubsection{Pressure}

We consider the adsorption isotherm at $80{ }^{\circ} \mathrm{C}$ in a 3.8 -nm-wide channel as a typical case. Fig. 6 a shows the obtained density profiles at different pressures from MD simulations. Fig. $6 \mathrm{~b}$ shows the corresponding excess and absolute isotherms, and these are denoted by red and black lines, respectively. The absolute isotherms calculated by the

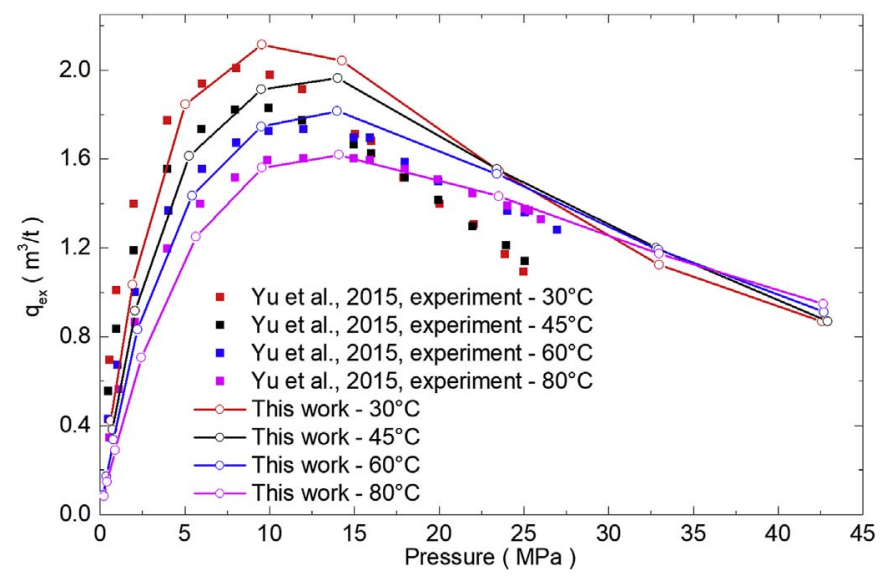

Fig. 5. Comparison of excess isotherms obtained by experiments and simulations. 
a)

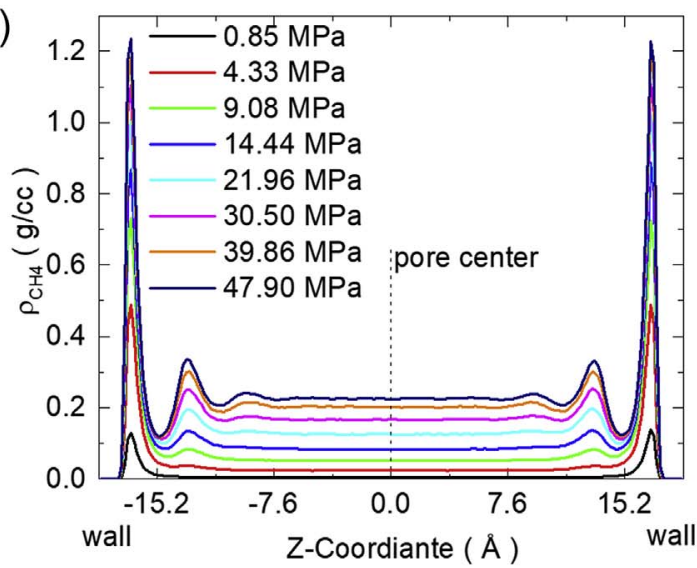

b)

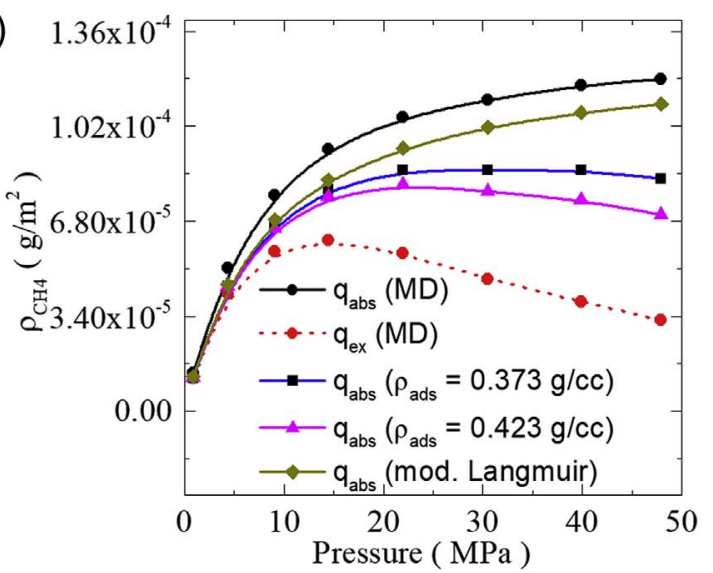

Fig. 6. (a) Density profiles under different pressures. (b) Excess and absolute adsorption isotherms obtained by different methods.

methods that assume that the adsorbed-phase density is fixed at $0.373 \mathrm{~g} / \mathrm{cc}$ (blue line) and $0.423 \mathrm{~g} / \mathrm{cc}$ (magenta line) both decline at high pressures and they appear unreasonable in describing physical adsorption. Therefore, the first category of methods may lead to completely false results, and they are so unstable that we do not further discuss these methods. With the modified Langmuir equation method, the absolute amount increases with pressure. However, it is lower than the criterion (the MD simulated result), and underestimates the actual adsorption amount. The relative error $\delta$ between the result from the modified Langmuir equation method and that from MD simulation was determined as

$\delta=\frac{q_{a b \text {.mod.Langmuir }}-q_{a b M D}}{q_{a b M D}} \times 100 \%$

The value of $\delta$ varies between $-11.75 \%$ and $-7.54 \%$ when the pressure increases from $0.85 \mathrm{MPa}$ to $47.90 \mathrm{MPa}$. The actual adsorbed-phase density (proportional to the absolute adsorption amount per unit surface area) increases with pressure, and the fitted adsorbed-phase density for the modified Langmuir equation is a constant of $0.321 \mathrm{~g} / \mathrm{cc}$. Because the actual adsorbed-phase densities are always smaller than the fitted one, according to Eq. (1), the absolute amount in the whole pressure range is always underestimated.

\subsubsection{Temperature}

In addition to the $80{ }^{\circ} \mathrm{C}$ condition, we also include adsorption isotherms at $20{ }^{\circ} \mathrm{C}$ for comparison. The adsorption isotherms exhibit a greater magnitude at $20^{\circ} \mathrm{C}$. The three-parameter Töth equation is also considered in this discussion. Firstly, both the Langmuir and Töth equations are used to fit the absolute adsorption isotherms obtained by MD simulations under the two temperature conditions. At $80{ }^{\circ} \mathrm{C}$, the fitted curves exhibit a close consistency; while at $20{ }^{\circ} \mathrm{C}$, there exists a slight difference (Fig. 7a). However, the adjust $\mathrm{R}^{2}$ values are all larger than 0.99 for these four fittings, which means that the fittings are all fairly satisfactory; both equations appear suitable for the sample.

Both modified equation methods are therefore used to calculate absolute the isotherms from the excess isotherms obtained by MD simulation. At $80{ }^{\circ} \mathrm{C}$, the result from the modified Töth equation method is always underestimated. The relative errors lie between $-12.48 \%$ and $-11.26 \%$, and they are larger than those from the modified Langmuir equation method (Fig. $7 \mathrm{~b}$ ). At $20^{\circ} \mathrm{C}$, the fitted adsorbed-phase densities based on the modified Langmuir and Töth equation methods are $0.343 \mathrm{~g} / \mathrm{cc}$ and $0.348 \mathrm{~g} / \mathrm{cc}$, respectively. The modified Langmuir equation method underestimates the absolute amount at low pressures, and overestimates it at high pressures. The relative errors lie between $-5.72 \%$ and $3.81 \%$. In comparison, the modified Töth equation method underestimates the absolute amount with relative errors in the range of $-9.69 \%$ to $-0.63 \%$. In summary, the modified Töth equation method always underestimates the absolute amount relative to the modified Langmuir equation method at different temperatures. The discrepancies are larger at higher temperatures, and when the temperature is lower, the relative errors of these two methods become smaller.

\subsubsection{Channel width}

We next simulated methane adsorptions in channels of different widths. Fig. 8a shows the absolute isotherms at $80{ }^{\circ} \mathrm{C}$. When the width exceeds $3.04 \mathrm{~nm}$, the isotherms are almost coincident. Considering the discrepancies of the results based on conversion methods of the second category, in a smaller 1.14-nm-wide channel, both the modified Langmuir and Töth equation methods underestimate the absolute adsorption amount at high pressures and the relative errors can exceed $30 \%$ at $30 \mathrm{MPa}$ (Fig. 8b). The fitted adsorbed-phase densities obtained by these methods are $0.348 \mathrm{~g} / \mathrm{cc}$ (Langmuir) and $0.3378 \mathrm{~g} / \mathrm{cc}$ (Töth). These values are significantly larger than the actual adsorbed layer density under these conditions, and this leads to the underestimations. Therefore, it can be concluded that when the sample is dominated by pores smaller than $3.04 \mathrm{~nm}$, the relative errors of the existing methods are fairly large.

\subsubsection{Comprehensive analysis}

In our study, a series of MD simulations is performed to cover the common range of temperature, pressure and pore width of actual shale reservoirs. The two abovementioned modified methods are applied to the excess adsorption amount data from MD simulations to calculate the absolute amounts. The converted absolute isotherms are compared with the MD simulated ones to determine the relative errors. Fig. 9 shows the relative error isosurfaces of these two methods. The overall trends of these two methods are closely consistent, thereby indicating that the errors are larger for higher pressure, higher temperature, and smaller channel width. With the modified Langmuir method, the relative errors lie between $-40 \%$ and $5 \%$, and with the modified Töth method, the relative errors lie between $-45 \%$ and $-5 \%$. These results can remind researchers who use the two sets of methods about the uncertainty of the results. This uncertainty may underlie the existing discrepancies of the absolute adsorption amounts estimated by isothermal adsorption tests and desorption tests (Bustin, 2004).

In addition, it needs to be mentioned that the MD simulations are performed on a homogeneous surface. In reality, the pore surface may exhibit heterogeneity. For example, the fitted $n$ value of the shale sample (Yu et al., 2015) in Fig. A1 is 0.51 (significantly smaller than 1), which means that the sample is fairly heterogeneous. In this situation, the relative errors of different methods may be quite different (Fig. A1), and this aspect needs to be further investigated in the future.

These relative error distributions provide an overview of the possible discrepancies occurring when the existing conversion methods are 

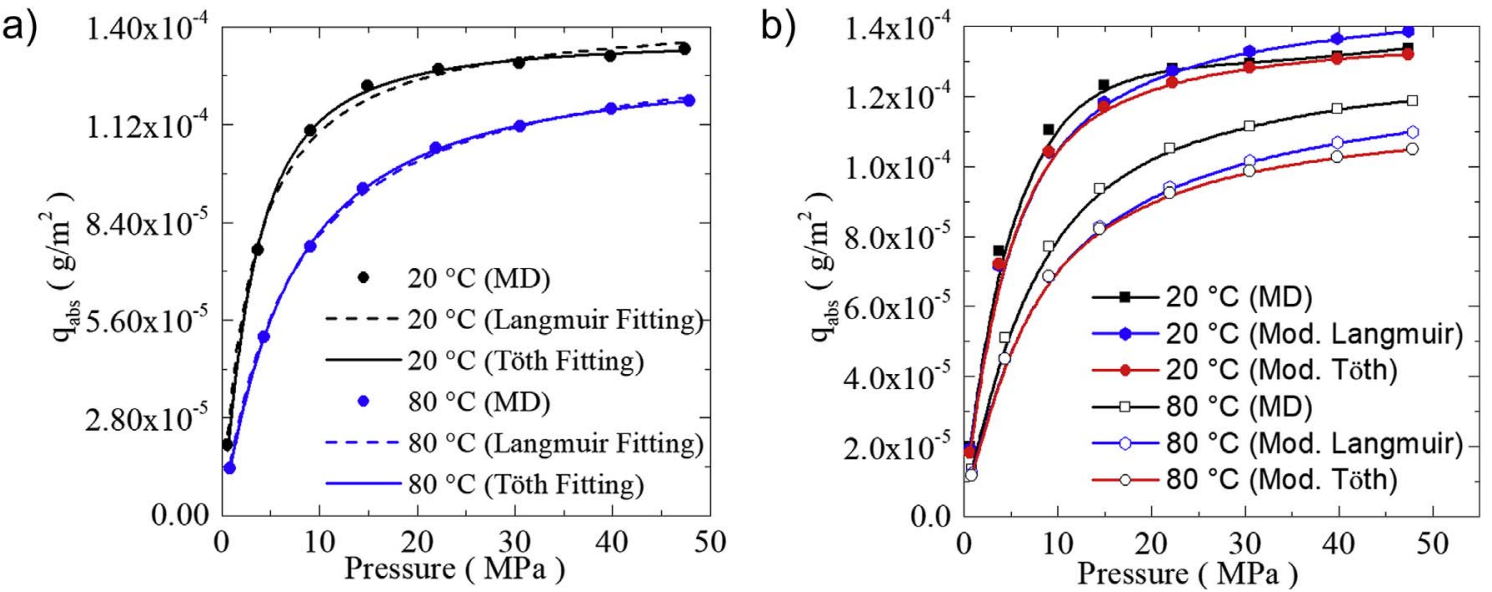

Fig. 7. Absolute adsorption isotherms under different temperatures as obtained by (a) molecular dynamics and (b) two modified methods.

applied to samples with similar excess adsorption isotherms. Even for the samples whose excess isotherms are different, the procedure proposed here can be suitable adopted as long as a reasonable MD model can be constructed.

\section{Conclusion}

In this study, we converted the excess isotherms of a shale sample from the Longmaxi Formation in Sichuan, China to absolute absorption isotherms using different existing methods. The results vary based on the methods applied, and this is significant because the uncertainties of these methods are poorly understood. Thus, it is imperative to find an approach that can reveal and evaluate the possible discrepancies. This study employed molecular dynamics (MD) to construct a simulation model that is able to reproduce the excess adsorption isotherms of the shale sample. Evaluations of existing conversion methods for excess adsorption amount were conducted based on MD simulation results. Sensitivity analyses were performed in nanometer channels of different widths under different temperature and pressure conditions. We also calculated the uncertainties of the results based on these methods.

We draw the following conclusions from the findings of our study:

It is feasible to develop a simplified MD model that is able to reproduce the excess adsorption isotherms of the shale sample considered in the study with acceptable accuracy. Simulations based on this model can further provide the absolute isotherms as criteria for evaluations of the existing conversion methods.

- The variation in the absolute adsorption amounts with channel widths can be determined by MD simulations. It is found that when the width exceeds $3 \mathrm{~nm}$, the absolute adsorption amount per unit surface area cannot increase anymore with channel width. For a homogeneous wall surface, the absolute isotherms at different temperatures can satisfactorily fit the Langmuir and Töth equations.

- For samples that exhibit excess adsorption isotherms similar to that of the sample studied here, the relative errors of the existing conversion methods are calculated. The methods of the first category that use predetermined density values between $0.373 \mathrm{~g} / \mathrm{cc}$ and $0.423 \mathrm{~g} / \mathrm{cc}$ usually underestimate the absolute amount at high pressures. The modified Langmuir equation method can overestimate or underestimate the results depending on the temperature, pressure, and channel-width conditions; while the modified Töth equation method always underestimates the absolute amount. The relative error ranges are determined in the study, and the relative error is found to be large at higher pressures, and temperatures in smaller channels.

\section{Acknowledgments}

This work is supported by the Major National Science and Technology Special Program of China (Grant No. 2017ZX05037-001), the National Program on Key Basic Research Project (973 Program, Grant No. 2014CB239004), the National Natural Science Foundation of China (Grant No. 41690132 and 41574129), and the Strategic Priority Research Program of the Chinese Academy of Sciences (Grant No. XDB10020302). Jiang gratefully thanks Dr. Sam Yang, Dr. Jiecheng Yang and Jie Lu for their assistance in revising the manuscript. a)

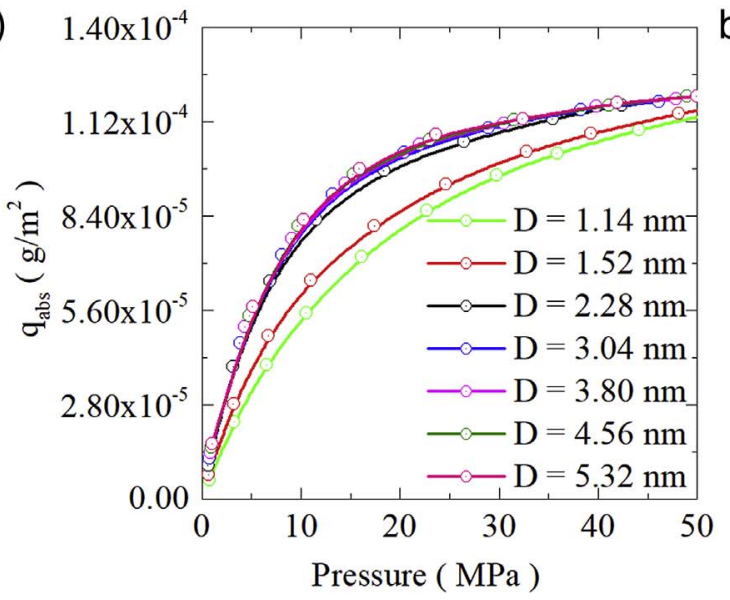

b)

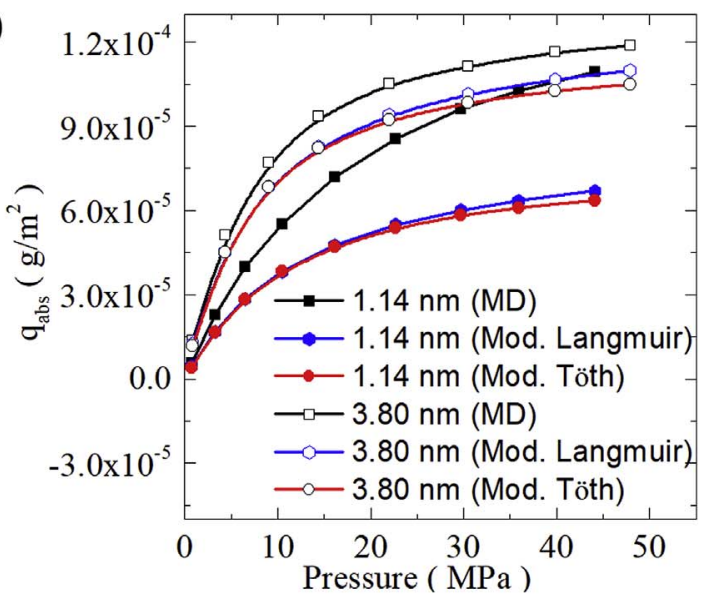

Fig. 8. Absolute adsorption isotherms of channels of different widths (a) obtained by molecular dynamics (MD). (b) those obtained by MD and two modified methods. 
a)
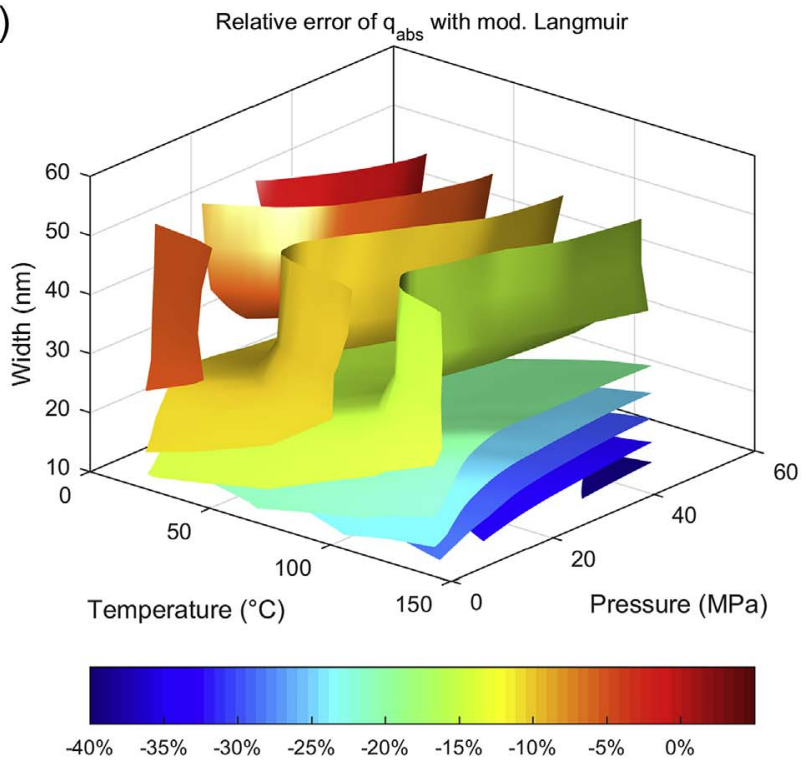

b)
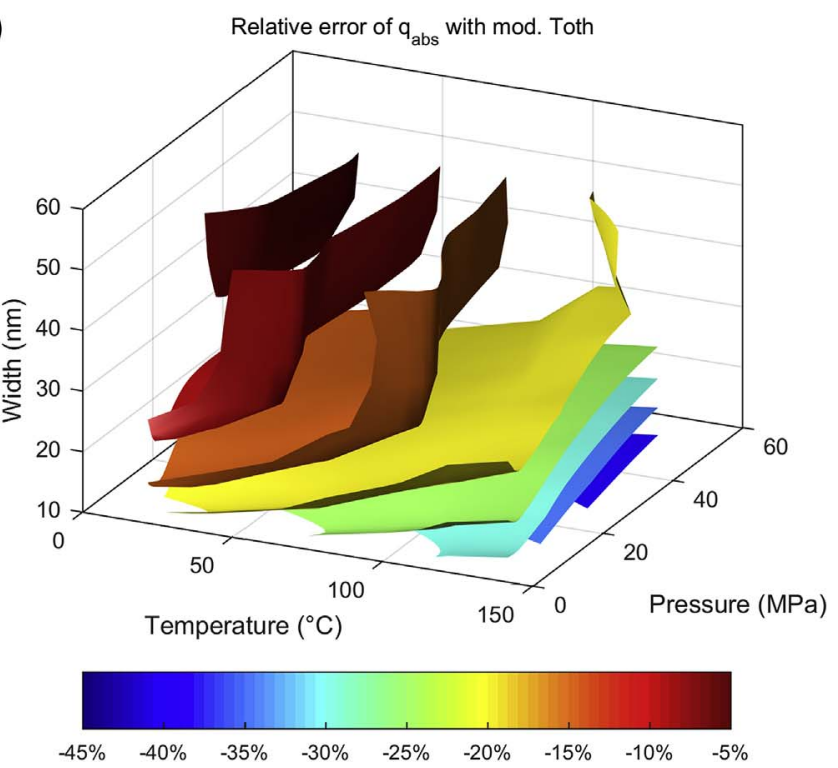

Fig. 9. Isosurfaces of relative errors of absolute adsorption amounts obtained by (a) the modified Langmuir equation method and (b) the modified Töth equation method.

\section{Appendix}

The $30{ }^{\circ} \mathrm{C}$ excess isotherm of a shale sample, of which the total organic content (TOC) is $4.52 \%$ and the porosity is $4.5 \%$, from the Longmaxi Formation in Sichuan, China (Yu et al., 2015) is converted to absolute isotherms using Eq. (1) with $\rho_{a d}$ given by the aforementioned methods. It can be observed that with methods of the first category, the absolute amounts decline with the pressure in the high pressure region, which appear unreasonable in describing physical adsorption (Fig. A1). With methods of the second category, when the data of the excess isotherms are fitted to the modified Langmuir and Töth equations, the adjusted $\mathrm{R}^{2}$ values of both fittings are 0.98 and 0.99 , respectively, thus indicating that both fittings are of high quality. However, the fitted $\rho_{a d}$ values obtained with these equations are different, and the discrepancy is about $10 \%$ (Table A3), and the converted absolute isotherms are obviously different (Fig. A2). 


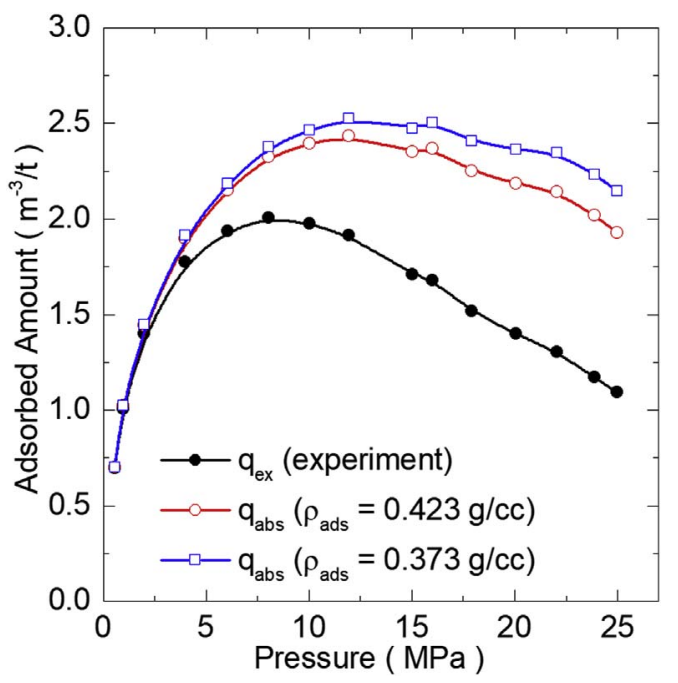

Fig. A1. Measured excess isotherm and converted absolute isotherms obtained with methods of the first category.

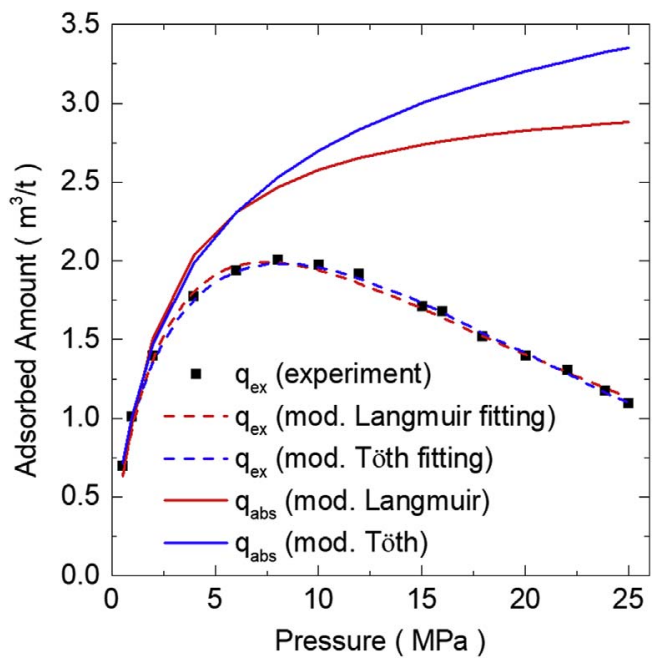

Fig. A2. Measured excess isotherm and converted absolute isotherms obtained with methods of the second category.

Table A3

Fitted results obtained with the two different methods.

\begin{tabular}{lll}
\hline Method & Mod. Langmuir & Mod. Töth \\
\hline$V_{L}$ or $V_{T}\left(\mathrm{~g} / \mathrm{cm}^{3}\right)$ & 3.13 & 5.14 \\
$P_{L}$ or $P_{T}(\mathrm{MPa})$ & 2.15 & 1.63 \\
$n$ & - & 0.51 \\
$\rho_{a d}(\mathrm{~g} / \mathrm{cc})$ & 0.30 & 0.27 \\
\hline
\end{tabular}

\section{References}

Ahmed, U., Meehan, D.N., 2016. Characteristics of unconventional oil and gas resources. In: Ahmed, U., Meehan, D.N. (Eds.), Unconventional Oil and Gas Resources: Exploitation and Development. CRC Press, Florida, USA, pp. 3-10.

Ambrose, R.J., Hartman, R.C., Diaz-Campos, M., Akkutlu, I.Y., Sondergeld, C.H., 2012 Shale gas-in-place calculations part i: new pore-scale considerations. SPE J. 17, 219-229. https://doi.org/10.2118/131772-PA.

Bustin, R.M., 2004. Characterising Gas Shales by Laboraotry Adsorption and Field Desorption Analyses. Presented at the 2004 Rocky Mountain Section AAPG Meeting (With Colorado Oil\&Gas Association). Denver, Colorado, USA, August 9-11, 2004.

Chareonsuppanimit, P., Mohammad, S.A., Robinson Jr., R.L., Gasem, K.A.M., 2012. Highpressure adsorption of gases on shales: measurements and modeling. Int. J. Coal. Geol. 95, 34-46. https://doi.org/10.1016/j.coal.2012.02.005.

Chen, C., 2016. Multiscale imaging, modeling, and principal component analysis of gas transport in shale reservoirs. Fuel 182, 761-770. https://doi.org/10.1016/j.fuel. 2016.06.020.
Chen, C., Hu, D.D., Westacott, D., Loveless, D., 2013. Nanometer-scale characterization of microscopic pores in shale kerogen by image analysis and pore-scale modeling. Geochem. Geophy. Geosy 14, 4066-4075. https://doi.org/10.1002/ggge.20254.

Civan, F., Rai, C.S., Sondergeld, C.H., 2010. Shale-gas permeability and diffusivity inferred by improved formulation of relevant retention and transport mechanisms. Transp. Porous Med. 86, 925-944. https://doi.org/10.1007/s11242-010-9665-x.

Clarkson, C.R., Haghshemas, B., 2013. Modeling of supercritical fluid adsorption on organic-rich shale and coal. In: Presented at the SPE Unconventional Resources Conference-USA, the Woodlands, Texas, USA, April 10-12, . https://doi.org/10. 2118/164532-MS.

Cui, X., Bustin, A.M.M., Bustin, R.M., 2009. Measurements of gas permeability and diffusivity of tight reservoir rocks: different approaches and their applications. Geofluids 9, 208-223. https://doi.org/10.1111/j.1468-8123.2009.00244.x.

Curtis, M.E., Sondergeld, C.H., Ambrose, R.J., Rai, C.S., 2012. Microstructural investigation of gas shales in two and three dimensions using nanometer-scale resolution imaging. AAPG Bull. 96, 665-677. https://doi.org/10.1306/08151110188.

Gao, B., 2016. Geochemical characteristics and geological significance of shale gas from the lower Silurian Longmaxi Formation in Sichuan Basin, China. J. Nat. Gas 
Geoscience 1, 119-129. https://doi.org/10.1016/j.jnggs.2016.03.002.

Gasparik, M., Ghanizadeh, A., Bertier, P., Gensterblum, Y., Krooss, B.M., 2012. Highpressure methane and sorption isotherms of black shale from The Netherlands. Energ. Fuel 26, 4995-5004. https://doi.org/10.1021/ef300405g.

Hall, F.E., Zhou, C.H., Gasem, K.A.M., Robinson, J.R.L., Dan, Y., 1994. Adsorption of pure methane, nitrogen, and carbon dioxide and their binary mixtures on wet Fruitland coal. In: Presented at the SPE Eastern Regional Meeting, Charleston, West Virginia, November 8-10, . https://doi.org/10.2118/29194-MS.

Ji, L.M., Zhang, T.W., Milliken, K.L., Qu, J.L., Zhang, X.L., 2012. Experimental investigation of main controls to methane adsorption in clay-rich rocks. Appl. Geochem 27, 2533-2545. https://doi.org/10.1016/j.apgeochem.2012.08.027.

Li, D.L., Xu, C.Y., Wang, J.Y., Lu, D.T., 2014a. Effect of Knudsen diffusion and Langmuir adsorption on pressure transient response in shale gas reservoir. J. Pet. Sci. Eng. 124, 146-154. https://doi.org/10.1016/j.petrol.2014.10.012.

Li, Y.S., Teng, S.N., Shi, J.T., Wang, H., Wu, L., Teng, S., 2014b. A nano-pore scale gas flow model for shale gas reservoir. In: Presented at the SPE Biennial Energy Conference, Trinidad and Tobago, Spain, June 9-11, . https://doi.org/10.2118/ 169939-MS.

Li, Z.Z., Min, T., Chen, L., Kangd, Q.J., He, Y.L., Tao, W.Q., 2016. Investigation of methane adsorption and its effect on gas transport in shale matrix through microscale and mesoscale simulations. Int. J. Heat. Mass Tran 98, 675-686. https://doi.org/10. 1016/j.ijheatmasstransfer.2016.03.039.

Liu, P.L., Feng, Y.S., Zhao, L.Q., Li, N.Y., Luo, Z.F., 2015. Technical status and challenges of shale gas development in Sichuan Basin, China. Petroleum 1, 1-7. https://doi.org/ 10.1016/j.petlm.2015.03.001.

Liu, Y., Zhu, Y.M., Li, W., Xiang, J.H., Wang, Y., Li, J.H., Zeng, F.G., 2016. Molecular simulation of methane adsorption in shale based on grand canonical Monte Carlo method and pore size distribution. J. Nat. Gas. Sci. Eng. 30, 119-126. https://doi. org/10.1016/j.jngse.2016.01.046.

Loucks, R.G., Reed, R.M., Ruppel, S.C., Jarvie, D.M., 2009. Morphology, genesis, and distribution of nanometer-scale pores in siliceous mudstones of the Mississippian Barnett shale. J. Sediment. Res. 79, 848-861. https://doi.org/10.2110/jsr.2009.092.

Lu, X.C., Li, F.C., Watson, A.T., 1995. Adsorption measurements in Devonian shales. Fuel 74, 599-603. https://doi.org/10.1016/0016-2361(95)98364-K.

Mosher, K., He, J.J., Liu, Y.Y., Rupp, E., Wilcox, J., 2013. Molecular simulation of methane adsorption in micro- and mesoporous carbons with applications to coal and gas shale systems. Int. J. Coal Geol. 109-110, 36-44. https://doi.org/10.1016/j.coal. 2013.01.001.

Mouahid, A., Bessieres, D., Plantier, F., Pijaudier-Cabot, G., 2012. Supercritical adsorption of nitrogen on EcoSorb-activated carbon at temperatures up to $383 \mathrm{~K}$ and pressures up to $2 \mathrm{MPa}$. J. Therm. Anal. Calorim. 109, 473-479. https://doi.org/10. 1007/s10973-011-1762-8.
Naraghi, M.E., Javadpour, F., 2015. A stochastic permeability model for the shale-gas systems. Int. J. Coal Geol. 140, 111-124. https://doi.org/10.1016/j.coal.2015.02. 004.

Nelson, P.H., 2009. Pore-throat sizes in sandstones, tight sandstones, and shales. AAPG Bull. 93, 329-340. https://doi.org/10.1306/10240808059.

Plimpton, S., 1995. Fast parallel algorithms for short-range molecular dynamics. J. Comput. Phys. 117, 1-19. https://doi.org/10.1006/jcph.1995.1039.

Rexer, T.F.T., Benham, M.J., Aplin, A.C., Thomas, K.M., 2013. Methane adsorption on shale under simulated geological temperature and pressure conditions. Energy Fuel 27, 3099-3109. https://doi.org/10.1021/ef400381v.

Rexer, T.F.T., Mathia, E.J., Aplin, A.C., Thomas, K.M., 2014. High-pressure methane adsorption and characterization of pores in Posidonia shales and isolated kerogens. Energy Fuel 28, 2886-2901. https://doi.org/10.1021/ef402466m.

Santos, J.M., Akkutlu, I.Y., 2013. Laboratory measurement of sorption isotherm under confining stress with pore-volume effects. SPE J. 18, 924-931. https://doi.org/10 2118/162595-PA.

Sharma, A., Namasani, S., Singh, J.K., 2015. Molecular simulation of shale gas adsorption and diffusion in inorganic nanopores. Mol. Simulat 41, 414-422. https://doi.org/10. 1080/08927022.2014.968850.

Sigal, R.F., Deepak, D., Civan, F., 2015. A review of the critical issues surrounding the simulation of tranport and storage in shale reservoirs. In: Rezaee, R. (Ed.), Fundamentals of Gas Shale Reservoirs. John Wiley \& Sons, Inc., New Jersey, USA, pp. 269. https://doi.org/10.1002/9781119039228.ch12.

Song, W., Yao, J., Li, Y., Sun, H., Zhang, L., Yang, Y., Zhao, J., Sui, H., 2016. Apparent gas permeability in an organic-rich shale reservoir. Fuel 181, 973-984. https://doi.org/ 10.1016/j.fuel.2016.05.011.

Sui, H.G., Yao, J., Zhang, L., 2015. Molecular simulation of shale gas adsorption and diffusion in clay nanopores. Computation 3, 687-700. https://doi.org/10.3390/ computation 3040687.

Toth, J.A., 1995. Various quantum mechanical aspects of quadratic forms. J. Funct. Anal. 130, 1-42. https://doi.org/10.1006/jfan.1995.1062.

Wu, H.A., Chen, J., Liu, H., 2015. Molecular dynamics simulations about adsorption and displacement of methane in carbon nanochannels. J. Phys. Chem. C 119, 13652-13657. https://doi.org/10.1021/acs.jpcc.5b02436.

Yang, F., Ning, Z.F., Kong, D.T., Peng, P., Zhao, H.W., 2013. Comparison analysis on model of methane adsorption isotherms in shales. Coal Sci. Technol. 41, 86-89.

Yu, L.J., Fan, M., Chen, H.Y., Liu, W.X., Zhang, W.T., Xu, E.S., 2015. Isothermal adsorption experiment of organic-rich shale under high temperature and pressure using gravimetric method. Acta Pet. Sin. 36, 557-563.

Zhang, T.W., Ellis, G.S., Ruppel, S.C., Milliken, K., Yang, R.S., 2012. Effect of organicmatter type and thermal maturity on methane adsorption in shale-gas systems. Org. Geochem 47, 120-131. https://doi.org/10.1016/j.orggeochem.2012.03.012. 